\title{
Determination of the maximum allowable distance between the roller conveyors of a tubular belt conveyor
}

\author{
Ruslan Kiriia ${ }^{1, *}$, and Tamara Mishchenko ${ }^{1}$ \\ ${ }^{1}$ Institute of Geotechnical Mechanics named by N. Poljakov of National Academy of Sciences of \\ Ukraine, 49005, Dnipro, Simferopolska Str., 2a, Ukraine
}

\begin{abstract}
The article developed a mathematical model of the stress-strain state of a tubular conveyor belt filled with bulk load. In this case, the belt is considered as a thin elastic inextensible cylindrical shell, and the bulk load in the belt is in the limit state. A system of differential equilibrium equations for a tubular belt with a bulk load with respect to forces and bending moments in a belt was obtained, which, when simplified, was reduced to a fourth-order differential equation for belt deflections. Based on this mathematical model, analytical dependencies of the deflections of the tubular conveyor belt on the parameters of the conveyor, the radius and properties of the belt, as well as the properties of the bulk load are obtained and analyzed. As a result, the maximum allowable distance between the roller supports of the tubular conveyor is determined. It was found that the allowable distance between the roller bearings is directly proportional to the tension of the belt and inversely proportional to the square of the radius of the belt and the bulk weight of the load. The research results can be used in the design of tubular belt conveyors transporting bulk load.
\end{abstract}

\section{Introduction}

Tubular belt conveyors are currently widely used in various industries: mining, metallurgy, construction and chemical. The main advantages of tubular conveyors, unlike ordinary belt conveyors with a grooved belt, are environmental friendliness and the ability to transport bulk load along curvilinear routes without overload devices. However, their effective use in industry is constrained by the lack of scientifically based methods for calculating the basic parameters of the tubular conveyor. In particular, there is no calculation of the maximum allowable distance between the roller supports of the tubular conveyor depending on the parameters of the conveyor, the elastic properties of the belt, as well as the physical and mechanical properties of the bulk load.

To solve this problem, it is necessary, first of all, to develop a mathematical model of the stress-strain state of a belt filled with a bulk load as it moves along the tubular conveyor roller supports.

\footnotetext{
*Corresponding author: kiriya_igtm@ukr.net
} 
Questions of creating a mathematical model of the stress-strain state of a belt filled with a bulk load were dealt with by V.G. Dmitriev, E.E. Sheshko, V.M. Gushchin, A.V. Dyachenko, D.S. Kulagin [1-3] and V.D. Chernenko [4]. In these works, the normal distributed forces of spreading bulk loads, acting on the belt from the bulk load as it moves along the roller conveyors, are investigated. At the same time the bulk load on the conveyor belt was in extreme equilibrium.

As a result, the analytical dependences of the distribution of these forces on the angular coordinates of the normal belt cross section were obtained. In this case, the deformation of the belt was not taken into account.

In the work of V.D. Chernenko, a mathematical model of the stress-strain state of a tubular belt filled with a bulk load was developed, based on the general theory of elastic shells and the limit state of a flowing medium. As a result, complex systems of partial differential equations were obtained, determining the strain stresses in the shell and the flowing medium, which for particular cases were solved by a numerical method.

In this paper, we developed a mathematical model of the stress-strain state of a tubular conveyor belt filled with a bulk load, based on the S. P. Timoshenko theory of thin elastic cylindrical shells. It was assumed that the shell is inextensible, and its deformations are small. As a result, analytical dependencies of the belt deflection on the parameters of the conveyor, the elastic properties of the belt, and the physical and mathematical properties of the bulk load are obtained. This relationship made it possible to determine the maximum allowable distance between the roller e support tubular belt conveyor.

\section{Methods}

Imagine a section of the tubular conveyor belt, located between the roller-supports, as a thin elastic cylindrical shell, clamped between the roller-supports (Fig. 1).

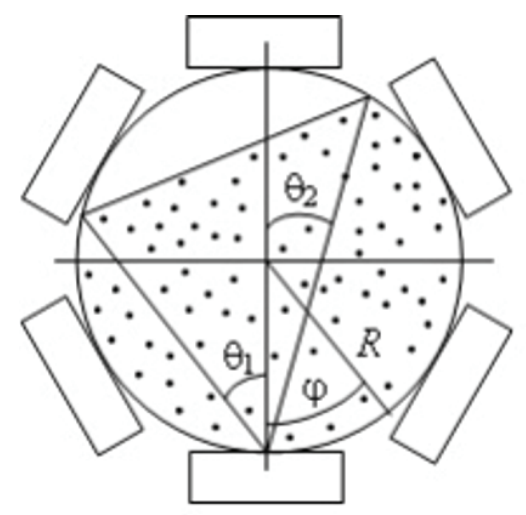

Fig. 1. Normal cross-section of a tubular conveyor belt.

In figure 1 marked: $\varphi$ is the current angular coordinate, rad; $\theta_{1}$ and $\theta_{2}$ are the angular coordinates of the intersection of the surface of the tubular section of the belt with a bulk load, rad.

According to the theory of thin elastic cylindrical shells $[5,6]$, forces and moments act on the element of the middle surface of the shell when it is deformed, and the deformation of the shell is considered to be essentially small compared to its radius $R(\mathrm{~m})$.

In this case, taking into account the simplifications of the system of equilibrium equations [5], after the elimination of the corresponding component equations, the system will have the form: 


$$
\left\{\begin{array}{l}
R \frac{\partial N_{x}}{\partial x}+\frac{\partial N_{\varphi x}}{\partial \varphi}=0 \\
\frac{\partial N_{\varphi}}{\partial \varphi}+R N_{x} \frac{\partial^{2} v}{\partial x^{2}}+R \frac{\partial N_{\varphi x}}{\partial x}-Q_{\varphi}+\tau R=0 \\
R \frac{\partial Q_{x}}{\partial x}+\frac{\partial Q_{\varphi}}{\partial \varphi}+R N_{x} \frac{\partial^{2} w}{\partial x^{2}}+N_{\varphi}+q R=0 \\
R \frac{\partial M_{x \varphi}}{\partial x}-\frac{\partial M_{\varphi}}{\partial \varphi}+R Q_{\varphi}=0 \\
\frac{\partial M_{\varphi x}}{\partial \varphi}+R \frac{\partial M_{x}}{\partial x}-R Q_{x}=0
\end{array}\right.
$$

As in [5], it is assumed: the $z$ axis is directed along the normal to the deformed middle surface of the conveyor belt, the $x$ axis is directed tangentially to the middle surface, the $y$ axis is directed perpendicular to the $x z$ plane; $N_{x}$ is the intensity of membrane tensile forces along the $x$-axis, $\mathrm{N} / \mathrm{m} ; N_{\varphi}$ is the intensity of the membrane forces in the cross section in the coordinate $\varphi, \mathrm{N} / \mathrm{m} ; N_{x \varphi}$ is intensity of tangential membrane forces, $\mathrm{N} / \mathrm{m} ; Q_{x}$ is intensity of shear forces in the direction of the $x$-axis, $\mathrm{N} / \mathrm{m} ; Q_{\varphi}$ is intensity of shear forces in the direction of the coordinates $\varphi, \mathrm{N} / \mathrm{m} ; M_{\varphi}, M_{x}, M_{x \varphi}, M_{\varphi x}$ are the intensities of bending and torsional moments of normal sections of a cylindrical shell element, $\mathrm{N} ; q$ is normal distributed force acting on the tape from the bulk load, $\mathrm{N} / \mathrm{m}^{2}$; and $\tau$ is shear stress acting on the conveyor belt from the side of bulk load, $\mathrm{N} / \mathrm{m}^{2}$.

We also consider small displacements of the shell points $u(\mathrm{~m}), v(\mathrm{~m})$ and $w(\mathrm{~m})$ respectively along the axis of symmetry $x$ of the belt shell, in the circumferential direction $\varphi$, and also along the normal to the surface $z$ of the belt shell.

Since the displacements $u, v, w$ are small, then, according to Hooke's law, the bending forces and moments are determined by the formulas $[5,6]$ :

$$
\begin{gathered}
N_{x}=\frac{E h}{1-v_{1}^{2}}\left(\varepsilon_{x}+v_{1} \varepsilon_{\varphi}\right) ; \quad N_{\varphi}=\frac{E h}{1-v_{1}^{2}}\left(\varepsilon_{\varphi}+v_{1} \varepsilon_{x}\right) ; N_{x \varphi}=\frac{\gamma_{x \varphi} E h}{2\left(1-v_{1}\right)} ; \\
M_{x}=-D\left(\chi_{x}+v_{1} \chi_{\varphi}\right) ; M_{\varphi}=-D\left(\chi_{\varphi}+v_{1} \chi_{x}\right) ; M_{x \varphi}=-M_{\varphi x}=D\left(1-v_{1}\right) \chi_{x \varphi},
\end{gathered}
$$

where

$$
\begin{gathered}
\varepsilon_{x}=\frac{\partial u}{\partial x} ; \quad \varepsilon_{\varphi}=\frac{\partial v}{R \partial \varphi}-\frac{w}{R} ; \quad \gamma_{x \varphi}=\frac{\partial u}{R \partial \varphi}+\frac{\partial v}{\partial x} \\
\chi_{x}=\frac{\partial^{2} w}{\partial x^{2}} ; \quad \chi_{\varphi}=\frac{1}{R^{2}}\left(\frac{\partial v}{\partial \varphi}+\frac{\partial^{2} w}{\partial \varphi^{2}}\right) ; \quad \chi_{x \varphi}=\frac{1}{R}\left(\frac{\partial v}{\partial x}+\frac{\partial^{2} w}{\partial x \partial \varphi}\right) .
\end{gathered}
$$

Here $\varepsilon_{x}$ is the deformation in the direction of the $\mathrm{x}$ axis; $\varepsilon_{\varphi}$ is deformation in the direction of $\varphi ; \gamma_{x \varphi}$ is angular deformation; $\chi_{x}$ is change of curvature in the direction of the $x$-axis, $\mathrm{m}^{-1} ; \chi_{\varphi}-$ change of curvature in the direction of the section, $\mathrm{m}^{-1} ; \chi_{x \varphi}$ is change of curvature in the direction of the section $x \varphi, \mathrm{m}^{-1} ; D$ is the cylindrical stiffness of the belt, $\mathrm{N} \cdot \mathrm{m} ; h$ is conveyor belt thickness, $\mathrm{m} ; E$ is elastic modulus of the belt material, $\mathrm{N} / \mathrm{m}^{2}$; and $v_{1}$ is Poisson ratio of the belt.

The cylindrical rigidity of a tubular belt according to [5] according to the formula $D=E h^{3} / 12\left(1-v_{1}^{2}\right)$. 
Given the fact that the longitudinal forces, i.e. the tension forces of the belt $N_{x}$ are many times greater than the lateral forces $N_{\varphi}$, they can be neglected, i.e. $N_{\varphi}=0$. Since the tape twisting forces are also absent, the moments from these forces are respectively zero, i.e. $M_{x \varphi}=M_{\varphi x}=0$. In addition, in our case, in addition to the tension forces $N_{x}$ acting on the belt, it is necessary to take into account the membrane tangential forces acting in the normal and longitudinal sections of the tubular conveyor belt, i.e. $N_{x} \neq 0 ; N_{x \varphi}=N_{\varphi x} \neq 0$.

Since $M_{x \varphi}=M_{\varphi x}=0$, then from the last two equations of system (1) there are equalities:

$$
Q_{\varphi}=\frac{1}{R} \frac{\partial M_{\varphi}}{\partial \varphi} ; Q_{x}=\frac{\partial M_{x}}{\partial x} .
$$

Substituting these equations in the second and third equations (1), then the system of equations (1) takes the form:

$$
\left\{\begin{array}{l}
R \frac{\partial N_{x}}{\partial x}+\frac{\partial N_{\varphi x}}{\partial \varphi}=0 \\
R N_{x} \frac{\partial^{2} v}{\partial x^{2}}+R \frac{\partial N_{\varphi x}}{\partial x}-\frac{1}{R} \frac{\partial M_{\varphi}}{\partial \varphi}+\tau R=0 \\
R \frac{\partial^{2} M_{x}}{\partial x^{2}}+\frac{1}{R} \frac{\partial^{2} M_{\varphi}}{\partial \varphi^{2}}+R N_{x} \frac{\partial^{2} w}{\partial x^{2}}+q R=0
\end{array}\right.
$$

Differentiating the second equation of system (4) by $\varphi$ and substituting the obtained equality into the third equation of this system, taking into account the first equation and equalities (3), after the transformation we get:

$$
R \frac{\partial^{2} M_{x}}{\partial x^{2}}+R N_{x} \frac{\partial^{3} v}{\partial x^{2} \partial \varphi}-R^{2} \frac{\partial^{2} N_{x}}{\partial x^{2}}+R N_{x} \frac{\partial^{2} w}{\partial x^{2}}+R \frac{\partial \tau}{\partial \varphi}+q R=0 .
$$

Assuming that the tape along the $x$ axis is not stretchable, therefore the tensile force $N_{x}$ does not depend on $x$ and $\varphi$, i.e. $N_{x}=s=$ const, then equation (5) after the transformation takes the form:

$$
\frac{\partial^{2} M_{x}}{\partial x^{2}}+s \frac{\partial^{3} v}{\partial x^{2} \partial \varphi}+s \frac{\partial^{2} w}{\partial x^{2}}+\frac{\partial \tau}{\partial \varphi}+q=0
$$

where $s$ is the intensity of tensile tensions $\left(s=S_{b} / B\right), \mathrm{N} / \mathrm{m} ; S_{b}$ is tension of the belt, $\mathrm{N}$; and $B$ is conveyor belt width $(B \approx 2 \pi R)$, $\mathrm{m}$. tension of the belt.

According to (2) we have:

$$
M_{x}=-D\left[\frac{\partial^{2} w}{\partial x^{2}}+\frac{v_{1}}{R}\left(\frac{\partial^{2} v}{\partial \varphi^{2}}+\frac{\partial^{2} w}{\partial \varphi^{2}}\right)\right] ; \quad M_{\varphi}=-D\left[\frac{1}{R}\left(\frac{\partial^{2} v}{\partial \varphi^{2}}+\frac{\partial^{2} w}{\partial \varphi^{2}}\right)+v_{1} \frac{\partial^{2} w}{\partial x^{2}}\right]
$$

From the last relations we get:

$$
M_{x}=-D\left(1-v_{1}^{2}\right) \frac{\partial^{2} w}{\partial x^{2}}+v_{1} M_{\varphi}
$$

Let us differentiate equality (7) twice in $x$ and substitute it in (6) taking into account the 
fact that $M_{\varphi}=$ const, i.e. assuming that the longitudinal bending moments in the belt do not depend on the $x$ coordinate, we obtain:

$$
D_{1} \frac{\partial^{4} w}{\partial x^{4}}-s \frac{\partial^{3} v}{\partial x^{2} \partial \varphi}-s \frac{\partial^{2} w}{\partial x^{2}}-\frac{\partial \tau}{\partial \varphi}-q(\varphi)=0 .
$$

where $D_{1}=D\left(1-v_{1}^{2}\right)$.

Suppose that the tensile deformation of the cross section of the belt is zero, that is, $\varepsilon_{\varphi}=0$. Then according to (2) we have:

$$
\varepsilon_{\varphi}=\frac{\partial v}{R \partial \varphi}-\frac{w}{R}=0
$$

From the last equality we have:

$$
\frac{\partial v}{\partial \varphi}=w
$$

Differentiating equality (9) twice in $x$ and substituting it in (8), we obtain the differential equation for the deflection of the conveyor belt:

$$
D_{1} \frac{\partial^{4} w}{\partial x^{4}}-2 s \frac{\partial^{2} w}{\partial x^{2}}-\frac{\partial \tau}{\partial \varphi}-q(\varphi)=0 .
$$

Since the belt is not supposed to be deformed in the radial and circumferential directions, i.e. is clamped, then the boundary conditions take the form:

$$
\left.w\right|_{x=0}=\left.w\right|_{x=l_{r}}=0 ;\left.\quad \frac{\partial w}{\partial x}\right|_{x=0}=\left.\frac{\partial w}{\partial x}\right|_{x=l_{r}}=0,
$$

where $l_{r}$ is the distance between the rollers, $\mathrm{m}$.

Assuming that the tangential stresses acting on the tape from the bulk load, obey the Amont-Coulomb law, we have:

$$
\tau=f_{1} q
$$

where $f_{1}$ is the coefficient of friction of the bulk load on the conveyor belt.

Equation (10) in view of (12) we write in the form:

$$
D_{1} \frac{\partial^{4} w}{\partial x^{4}}-2 s \frac{\partial^{2} w}{\partial x^{2}}-\frac{\partial \tau}{\partial \varphi}-q_{1}(\varphi)=0,
$$

where $q_{1}(\varphi)=f_{1} \frac{d q}{d \varphi}+q(\varphi)$.

According to [2], when the belt moves along the roller conveyors of the tubular conveyor, active and passive normal distributed loads, equal to: 


$$
\begin{aligned}
& q_{a}=R \gamma\left(\cos ^{2} \varphi+m \sin ^{2} \varphi\right)(\cos 2 \theta+\cos \varphi) \\
& q_{n}=R \gamma\left(\cos ^{2} \varphi+\frac{\sin ^{2} \varphi}{m}\right)(\cos 2 \theta+\cos \varphi),
\end{aligned}
$$

where $q_{a}$ is the active distributed load on the conveyor belt associated with its compression, $\mathrm{N} / \mathrm{m}^{2} ; q_{n}$ is passive distributed load on the conveyor belt, associated with its collapse, $\mathrm{N} / \mathrm{m}^{2} ; \varphi$ is the current angular coordinate in the cross section of the tubular conveyor belt, rad; $\theta$ is the angle that determines the degree of filling with a bulk load of the cross-section of the ribbon contour, rad (see Fig. 1); $m$ is the coefficient of mobility of the bulk load, $m=1+2 f^{2}-2 f \cdot\left(1+f^{2}\right)^{1 / 2}[7] ; f$ is coefficient of internal friction of bulk load; and $\gamma$ is the bulk density of the bulk load, $\mathrm{N} / \mathrm{m}^{3}$.

The average value of the normal distributed load on the belt from the bulk load is determined by the formula:

$$
q=\frac{q_{a}+q_{n}}{2}
$$

In the case of an asymmetrical arrangement of the bulk load in the cross section of the belt, we define $q$ by the known $q_{a}$ and $q_{p}$ separately for two sections of the circular section of the belt - the left $0 \leq \varphi \leq \pi-2 \theta_{1}$ and the right $-\left(\pi-2 \theta_{2}\right) \leq \varphi \leq 0$, where $\theta_{1}$ and $\theta_{2}$ are the degrees of filling of the left and right sides of the belt section, rad (see Fig. 1).

Substituting (14) into (15) for the left and right halves of the belt section, we obtain the average values of the distributed normal load $q$ on the conveyor belt on the part of the bulk load in the form:

$$
q(\varphi)=\left\{\begin{array}{l}
0,5 R \gamma\left(\cos 2 \theta_{1}+\cos \varphi\right)\left(2 \cos ^{2} \varphi+\frac{m^{2}+1}{m} \sin ^{2} \varphi\right) \quad \text { at } 0 \leq \varphi \leq \varphi_{1} \\
0,5 R \gamma\left(\cos 2 \theta_{2}+\cos \varphi\right)\left(2 \cos ^{2} \varphi+\frac{m^{2}+1}{m} \sin ^{2} \varphi\right) \text { at } \varphi_{2} \leq \varphi \leq 0
\end{array}\right.
$$

where $\varphi_{1}=\pi-2 \theta_{1} ; \varphi_{2}=-\left(\pi-2 \theta_{2}\right)$.

In the case of the absence of friction forces $(\tau=0)$, equation (10) takes the form:

$$
D_{1} \frac{\partial^{4} w}{\partial x^{4}}-2 s \frac{\partial^{2} w}{\partial x^{2}}-q(\varphi)=0 .
$$

Solving equation (17) taking into account the boundary conditions (11), we obtain the equation of deflections of the tubular conveyor belt without taking into account the friction forces of the bulk load on the conveyor belt [5]:

$$
w=\frac{q l_{r}^{2}}{4 u s \operatorname{sth} u} \cdot\left\{\frac{\operatorname{ch}\left[u\left(1-\frac{2 x}{l_{r}}\right)\right]}{\operatorname{ch} u}-1\right\}+\frac{q\left(l_{r}-x\right) x}{2 s},
$$


where $u=\frac{l_{r}}{2} \sqrt{\frac{s}{D_{1}}} ; s=\frac{S_{b}}{B} ; D_{1}=\frac{E h^{3}}{12}$.

Similarly, solving equation (10), taking into account the boundary conditions (11), we obtain the equation of the deflection of the tubular conveyor taking into account the friction forces of the bulk load on the conveyor belt $(\tau \neq 0)$ :

$$
w=\frac{q_{1} l_{r}{ }^{2}}{8 u^{\prime} \operatorname{sth}\left(u^{\prime}\right)}\left[\frac{\operatorname{ch}\left[u^{\prime}\left(1-\frac{2 x}{l_{r}}\right)\right]}{\operatorname{ch}\left(u^{\prime}\right)}-1\right]+\frac{q_{1}\left(l_{r}-x\right) x}{4 s},
$$

where $u^{\prime}=\frac{l_{r}}{2} \sqrt{\frac{2 s}{D_{1}}}$.

Analysis of formulas (18) and (19) showed that since $S_{b}>>D_{1}\left(u>>1, u^{\prime}>>1\right)$, the first terms in these formulas are an order of magnitude smaller than the second and can be neglected. As a result of the equation of deflection, the belt will look like

- in the absence of friction forces, i.e. $(\tau=0)$ :

$$
w=\frac{q\left(l_{r}-x\right) x B}{2 S_{b}} ;
$$

- in case of presence of friction forces, i.e. $(\tau \neq 0)$ :

$$
w=\frac{q_{1}\left(l_{r}-x\right) x B}{4 S_{b}} .
$$

In addition, if we imagine a belt with a load of a tubular conveyor in the form of a flexible thread loaded with a distributed load, then its deflection is determined by the formula [7]:

$$
w=\frac{q_{0}\left(l_{r}-x\right) x}{2 S_{b}},
$$

where $q_{0}$ is the maximum linear load on the conveyor belt $\left(q_{0}=\pi R^{2} \gamma\right), \mathrm{N} / \mathrm{m}$.

Suppose that the tubular conveyor belt is fully loaded with a bulk load. Then $\theta_{1}=\theta_{2}=0$, and the deflection at the lowest point of the tubular belt with $\varphi=0$ is determined by the formula (19), where $q=q_{1}=2 R \gamma$.

\section{Result and discussion}

From the analysis of formulas (20), (21) and (22), it follows that the deflections of the tubular conveyor belt without considering the friction forces are twice the deflections of the belt, taking into account the friction forces of the same tubular conveyor, and the deflections of the tubular conveyor belt taking into account the friction forces two times more deflections of a flexible thread loaded with the same distributed load as the tubular belt.

Figure 2 shows the graphs of the deflection of a belt of a tubular belt, loaded as much as possible with a bulk load in the absence of friction forces, i.e. $\tau=0$ (curve 1), and taking 
into account the friction forces, i.e. $\tau \neq 0$ (curve 2), and deflection of the thread loaded with the same distributed load as the tubular belt (curve 3), from the $x$ coordinate $\left(0 \leq x \leq l_{r}\right)$. The parameters of the belt and the properties of the load took the following values: $S_{b}=20000 \mathrm{~N} ; R=0.25 \mathrm{~m} ; h=0.01 \mathrm{~m} ; l_{r}=1 \mathrm{~m} ; E=2 \cdot 10^{7} \mathrm{~N} / \mathrm{m}^{2} ; \gamma \approx 10000 \mathrm{~N} / \mathrm{m}^{3}$.

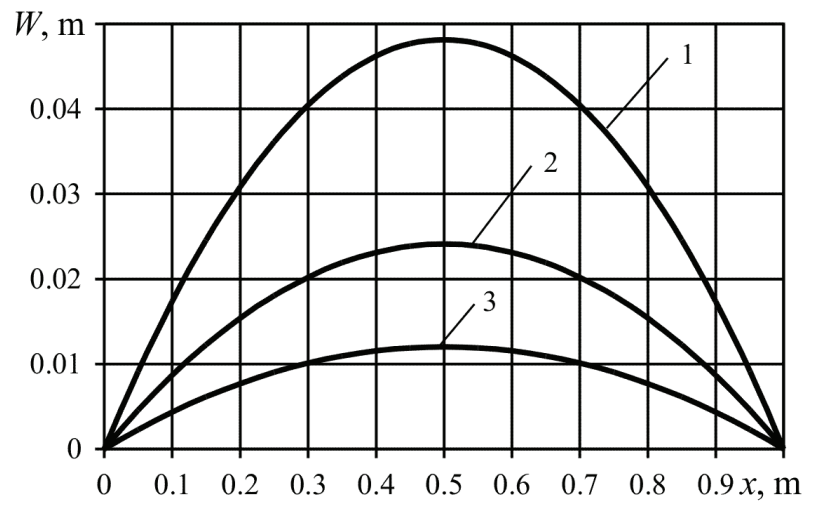

Fig. 2. Graph of the deflection of the belt on the x coordinate: 1 - tubular belt without friction; 2 tubular tape with regard to friction forces; 3 - flexible thread.

It can be seen from the graphs (Fig. 2) that the maximum deflection of the tubular belt and the flexible thread loaded with the same maximum distributed load is in the middle of the roller supports, i.e. at $x=l_{r} / 2$.

Based on the results of analytical studies, we will now determine the allowable distance between the roller supports of the tubular conveyor.

Substituting in (20) - (22) $x=l_{r} / 2$, we obtain the maximum deflections of the tubular belt and the flexible yarn loaded with the same distributed load as the tubular belt:

- tubular belt without friction

$$
w_{1 \max }=\frac{\pi R^{2} \gamma l_{r}^{2}}{2 S_{b}}
$$

- a tubular belt taking into account friction forces

$$
w_{2 \max }=\frac{\pi R^{2} \gamma l_{r}^{2}}{4 S_{b}} ;
$$

- flexible thread

$$
w_{3 \max }=\frac{\pi R^{2} \gamma l_{r}^{2}}{8 S_{b}} .
$$

It follows from formulas (23) - (25) that the maximum deflection of a tubular belt, completely filled with a bulk load, in the absence of friction forces of a bulk load on the belt is twice the maximum deflection of the tubular belt, taking into account the friction forces and four times the maximum deflection flexible yarn loaded with the same distributed maximum running load as the tubular conveyor belt.

According to [8], the allowable maximum deflection of the belt $f_{d}$ is in the range $f_{d}=(0.0125-0.025) l_{r}$ or

$$
f_{d}=k_{p} l_{r}
$$


where $k_{p}=0.0125-0.025$.

Substituting in formulas $(23)-(25)$ instead of $w_{1 \max }, w_{2 \max }, w_{3 \max } f_{d}$ from expression (26) and determining from the obtained equalities $l_{r}$, we obtain the maximum allowable distance between the roller supports for:

- tubular belt without friction

$$
l_{1 \max }=\frac{2 S_{b} k_{p}}{\pi R^{2} \gamma}
$$

- tubular tape taking into account friction forces

$$
l_{2 \max }=\frac{4 S_{b} k_{p}}{\pi R^{2} \gamma} ;
$$

- flexible thread

$$
l_{3 \max }=\frac{8 S_{b} k_{p}}{\pi R^{2} \gamma} .
$$

Putting $S_{b}=20000 \mathrm{~N} ; R=0.25 \mathrm{~m} ; \gamma \approx 10000 \mathrm{~N} / \mathrm{m}^{3} ; k_{p}=0.025$, according to formulas (27) - (29) we have

$$
l_{1 \max } \approx 0.5 \mathrm{~m} ; l_{2 \max } \approx 1.0 \mathrm{~m} ; l_{3 \max } \approx 2.1 \mathrm{~m}
$$

\section{Conclusions}

1. Developed a mathematical model of the stress-strain state filled with a bulk load belt as it moves along the roller of the tubular conveyor. In this case, the belt is a thin elastic cylindrical inextensible shell, and the bulk load is in the limit state.

2. Analytical dependences of the deflections of the tubular conveyor belt on the tension and radius of the belt, its elastic properties, the distance between the roller supports, and the physicomechanical properties of the bulk load are obtained. It was found that the maximum deflection of a tubular belt, completely filled with a bulk load, in the absence of friction forces of a bulk load on the belt, is twice the maximum deflection of the tubular belt, taking into account the friction forces, and four times the maximum deflection of the flexible thread loaded with the same distributed maximum running load as the tubular conveyor belt.

3. Based on the obtained dependency, the maximum allowable distance between the roller supports of the tubular conveyor is determined. It was determined that the maximum allowable distance between the roller supports is proportional to the tension of the belt, inversely proportional to the square of the radius of the belt and the volume weight of the transported load.

\section{References}

1. Dmitriev, V.G., Kulagin, D. S. (2004). Design of the stress state of tubular conveyer belt. Gornyy informatsionno-analiticheskiy byulleten, (7), 283-286

2. Sheshko, E. E., Gushin, V. M. (1973). Steeply inclined conveyor belt, which has form of deep troughs. Razvitie $i$ sovershenstvovanie shachtnogo i kariernogo transporta. Moskva: Nedra 
3. Dmitriev, V.G., Dyachenko, V. P. (2004). Methods of analysis of the volume stress state of bulk load in the closed groove of tubular band conveyer. Gornyy informatsionnoanaliticheskiy byulleten, (12), 241-243

4. Chernenko, V.D. (1985). Teoriya i raschet krutonaklonnykh konveyerov. Leningrad: Izdatelstvo LGU

5. Timoshenko, S.P., Voynovskiy-Kriger, S. (1963). Plastiny i obolochki. Moskva: Fizmatlit

6. Vlasov, V.Z. (1962). Obshchaya teoriya obolochek $i$ eye prilozheniya $v$ tekhnike. Moskva: Izdatelstvo Akademii nauk SSSR

7. Birger, I. A. (1986). Soprotivlenie materialov. Uchebnoe posobie. Moskva: Nauka

8. Shakhmeyster, L.G., Dmitriev, V.G. (1987). Teoriya i raschet lentochnykh konveyerov. Moskva: Mashinostroyeniye 